\title{
Corrective Day-ahead Prediction of Solar Radiation based on Online Measurements
}

\author{
Y.Kobayashi ${ }^{1}$,R.Watanabe ${ }^{1}$ Y.Hida $^{1}$, and R. Yokoyama ${ }^{1}$ \\ T.Funabashi ${ }^{2}$ \\ ${ }^{1}$ Graduate School of Environment and Energy Engineering \\ Waseda University \\ Shinjyuku-ku, Tokyo 169-8555, Japan \\ Phone/Fax number: +81-3-86-3126, e-mail: y-koba@fuji.waseda.jp \\ ${ }^{2}$ Meidensha Corporation \\ ThinkPark Tower, 2-1-1 Ohsaki, Shinagawa-ku, \\ Tokyo 141-6029 Japan \\ tel: $+81-3-6420-7208$ \\ fax: +81-3-5745-3042 \\ e-mail:funabashi-t@mb.meidensha.co.jp
}

\begin{abstract}
In recent years, reduction of greenhouse gas emissions has been a serious issue throughout the world. The Japanese Government aims to reduce $25 \%$ of the emissions in 1990 by 2020 . Eco-cities which effectively use renewable energy sources attract an attention in order to attain this goal. A smart energy town project in Honjyo, Japan has been conducted by making use of renewable energy sources. Above all, photovoltaic (PV) generators are expected to play a key role in this project. In addition, PV generators are expected to be installed in the residential and commercial areas due to ease of its installation. However, unstable power output from PV generation causes negative impacts on power quality of distribution networks. Therefore, prediction of PV output is indispensable to operate networks properly. In this paper, we propose a method to predict a day-ahead solar radiation and output power of PV generation in specific areas. Furthermore, the predicted solar radiation is corrected using the on-line measured actual data. As a result, the correction of a day-ahead prediction proposed here reduces errors of daily solar radiation by around $34 \%$.
\end{abstract}

\section{Key words}

Photovoltaic generators, Solar Radiation Forecast, Weather forecast

\section{Introduction}

Renewable energy sources such as solar and wind power generation are expected to play an important role to reduce $\mathrm{CO}_{2}$ emissions against global warming. Regional energy networks which contains these generators have been demonstrated all over the world. Although photovoltaic generators (PV) are most promising of all renewable energy in Japan, the generated power fluctuates due to intermittent solar radiation. Its fluctuation causes the imbalance between demand and supply and deteriorates power quality in distribution networks.
Therefore, an efficient prediction of solar radiation is required to develop for operating networks properly.

In Japan, weather observation stations for Automated Meteorological Data Acquisition System (AMeDAS) are located in several cities. The prediction method of solar radiation utilizing these extensive data has been reported in the previous studies. However, there is an issue to be solved in conventional studies. Since weather data is measured only in major cities by AMeDAS, it is difficult to obtain the detail regional weather data for the prediction of solar radiation in an individual area.

As a solution for this issue, an effective prediction method is proposed using the data which is measured online and is easy to esquire. The main procedures of this method are shown as follows;

(1) Weather conditions are classified into 6 and 15 patterns and weather forecasts are made.

(2) Predictions of a day-ahead solar radiation is carried out based on the weather forecasts.

(3) A day-ahead prediction is corrected using the online measured data on the day.

Furthermore, the performance of the proposed method is evaluated.

\section{Outline of the experimental installation and solar radiation analysis}

Actual meteorological data have been measured and recorded at Honjyo campus of Waseda University for three years (2009 2011). The measured items consisit of average wind speed $[\mathrm{m} / \mathrm{s}]$, average wind direction, pressure [hpa], temperature [degree], humidity [\%], solar radiation $\left[\mathrm{kW} / \mathrm{m}^{2}\right]$, and the maximum instantaneous wind speed $[\mathrm{m} / \mathrm{s}]$. These data are measured every minute. 


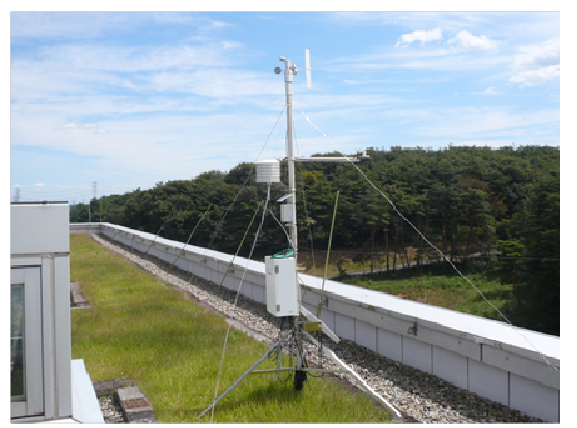

Fig. 1 Meteorological observation equipment at the Honjo campas of Waseda University,Japn

Table 1 shows the specification of the meteorological observation equipment and Fig. 2 shows the duration curve in which the accumulated solar radiations of the day are sorted in the descending order.

Table1. Specification of insolation sensor

\begin{tabular}{|c|c|}
\hline Wavelength & $305 \sim 2800[\mathrm{~nm}]$ \\
\hline Sensitivity & $7\left[\mathrm{mV} / \mathrm{kW} \cdot \mathrm{m}^{-2}\right]$ \\
\hline Accuracy & $\pm 3[\%]$ \\
\hline Internal resistance & $5 \sim 10[\mathrm{k} \Omega]$ \\
\hline
\end{tabular}

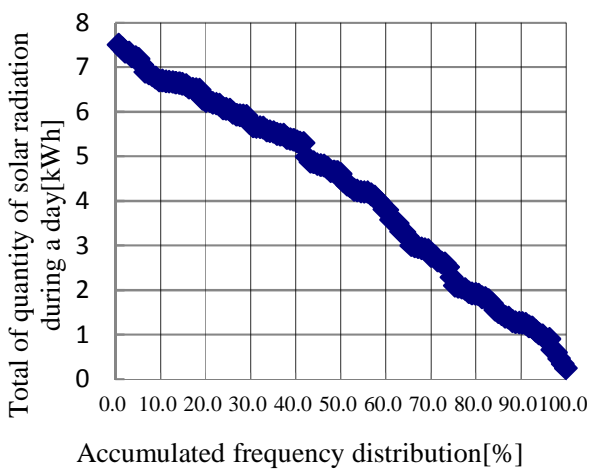

Fig. 2 Duration curve of radiation energy per day

\section{Prediction of a day-ahead solar radiation \\ 3.1 A day-ahead prediction of solar radiation}

The prediction is carried out every hour from 6:00 to 19:00. The prediction method is performed as follows.

(1) The recorded data are classified into 6 or 15 patterns as in table 2 or 3 by referring the actual past weather patterns of Japan Meteorological Agency.

(2) The monthly prediction curve is obtained by averaging the solar radiation in each pattern.

(3) The prediction curve is selected depending on the weather forecast on the day.

Moreover, hourly predicted values are calculated from 6:00 through 19:00. Table 2 and Table 3 show the weather patterns. The accuracy of predictions is expected to improve by reducing the number of weather patterns since the number of recorded data increases by reducing the number of patterns as in Table 2 and Table 3. The representative prediction curves of solar radiation, when the weather category is classified into six patterns, are shown in Fig. 3 and Fig. 4.
Table2. Classification of weather (15 patterns)

\begin{tabular}{|c|c|c|c|c|c|}
\hline Pattern & Weather & Pattern & Weather & Pattern & Weather \\
\hline 1 & clear,sunny & 6 & cloudy with occasional sunny & 11 & rain with occasional sunny \\
\hline 2 & sunny with occasional cloudy & 7 & cloudy & 12 & rain sunny later \\
\hline 3 & sunny cloudy later & 8 & cloudy sunny later & 13 & rain cloudy later \\
\hline 4 & sunny with occasional rain & 9 & cloudy with occasional rain & 14 & rain with occasional cloudy \\
\hline 5 & sunny rain later & 10 & cloudy rain later & 15 & rain,snow \\
\hline
\end{tabular}

Table3. Classification of weather (6 patterns)

\begin{tabular}{|c|c|c|c|}
\hline Pattern & Weather & Pattern & Weather \\
\hline 1 & clear,sunny & 4 & $\begin{array}{c}\text { sunny with occasional cloudy } \\
\text { sunny cloudy later }\end{array}$ \\
\hline 2 & $\begin{array}{c}\text { cludy,cloudy with occasional rain } \\
\text { cloudy rain later }\end{array}$ & 5 & $\begin{array}{c}\text { rain with occasional sunny } \\
\text { rain sunny later }\end{array}$ \\
\hline 3 & $\begin{array}{c}\text { rain,rain with occasional cloudy } \\
\text { rain cloudy later }\end{array}$ & 6 & $\begin{array}{c}\text { sunny with occasional rain } \\
\text { sunny rain later }\end{array}$ \\
\hline
\end{tabular}

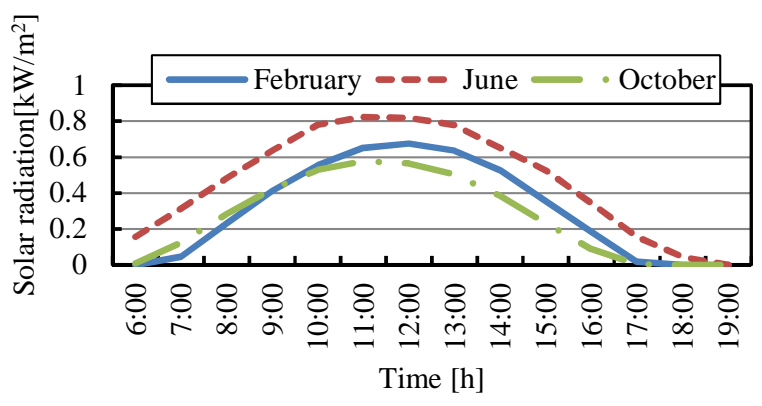

Fig. 3 The prediction curve of solar radiation in the weather pattern-1

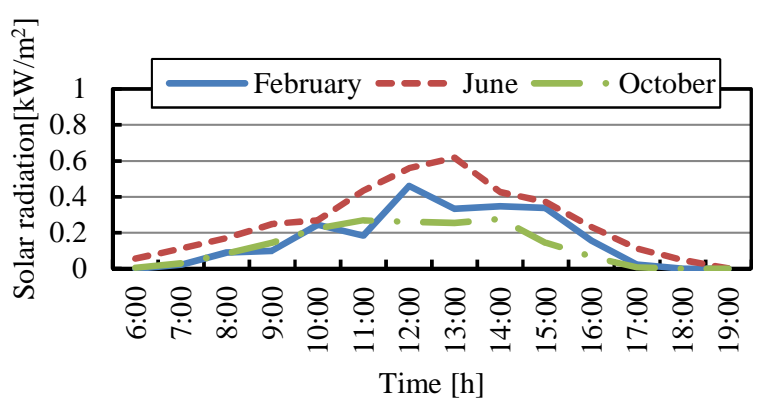

Fig. 4 The prediction curve of solar radiation in the weather pattern-5

The seasonal fluctuation of solar radiation can be taken into consideration due to calculation of the monthly prediction curves in Fig. 3 and Fig. 4. From Fig. 4, we can see that the time period when solar radiation starts to go down differes in each month. The variation in solar radiation is not always match between the predictied curve and the online measured curve. Therefore, it is necessary to correct the prediction curve using the measured value of the day.

\subsection{Correction of the prediction curve on the day}

The fluctuation of actual solar radiation may be different from that of the forecasted radiation. Furthermore, the weather forecast provided by Japan Meteorological Agency is not always accurate. In this case, it is necessary to correct the prediction curve using the online measured data of the day. Furthermore, the correction is required to conduct in an early morning for operating energy-related facilities. Therefore, Table 4 shows the correlation of the solar radiation from 6:00 to 10:00 and the accumulated solar radiation on the targeted day. 
Table4. Correlation of the solar radiation of 6:00 to 10:00 and the accumulated solar radiation

\begin{tabular}{|c|c|c|c|c|c|}
\hline Time[h] & 6 & 7 & 8 & 9 & 10 \\
\hline $\begin{array}{c}\text { Correlation } \\
\text { coefficient }\end{array}$ & 0.62 & 0.75 & 0.83 & 0.88 & 0.87 \\
\hline
\end{tabular}

Table 4 shows that the correlation of the measured value after 8:00 and the accumulated solar radiation is high. Therefore, the correction should be worked out in the early morning to use the prediction for the facility operation. The online measured value at $8: 00$ is normalized by dividing by the predicted value at 8:00. The normalized coefficient $\mathrm{f}$ is used to correct the predicted value after 9:00 by multiplication to match with the measured value. Fig. 5 shows the correction of the prediction curve.

$$
f=\frac{G_{m}}{G_{p}}
$$

$G_{m}:$ the measured value of 8:00

$G_{p}$ : the predicted value of 8:00

$f$ :the ratio of the measured value and the predicted value

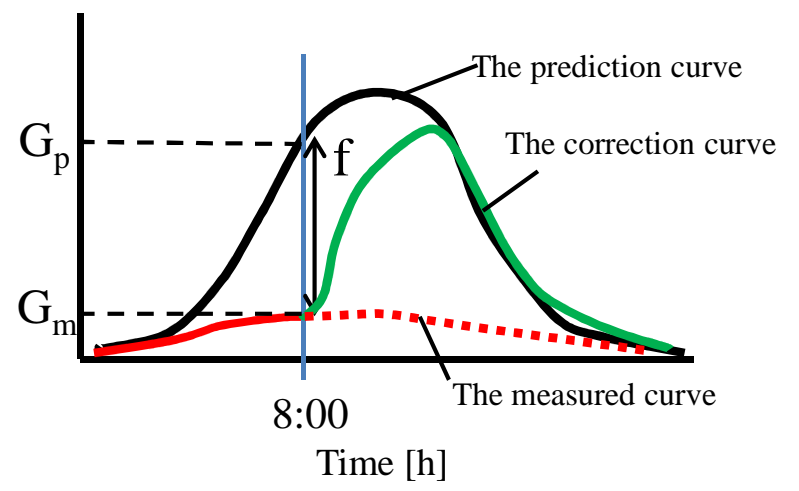

Fig. 5 Relation of various prediction curves

\section{Application to a practical test network}

The proposed online corrective prediction method is applied to a model test network by utilizing the measured data in 2012. In order to evaluate the accuracy of the prediction quantitatively, the error is defined as E. E can be expressed as follows;

$$
E\left[\mathrm{~kW} / \mathrm{m}^{2}\right]=\frac{\sum_{i}\left|G_{e s t i}-G_{m s r i}\right|}{n}
$$

$G_{e s t}:$ the prediction value of the solar radiation $\left[\mathrm{kW} / \mathrm{m}^{2}\right]$, $G_{m s r}$ : the measured value of the solar radiation $\left[\mathrm{kW} / \mathrm{m}^{2}\right]$, $i$ : time $[\mathrm{h}](6.00 \mathrm{am} \sim 7.00 \mathrm{pm}), n$ : the number of data

\subsection{Comparison of prediction erros of 6 and 15 weather patterns before correction}

Fig. 6 shows daily errors of the predicted values and the measured values for 6 and 15 weather patterns. It shows that errors are comparatively small except for that in the weather pattern-4. Fig. 7 shows comparisons of the predicted values and the measured value presented in Table 3 for the weather pattern- 4 .

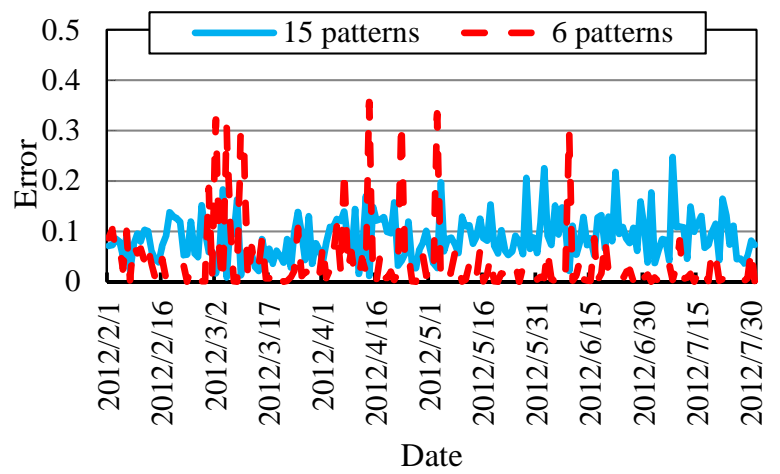

Fig. 6 Error for each day in the 6 patterns and 15 patterns

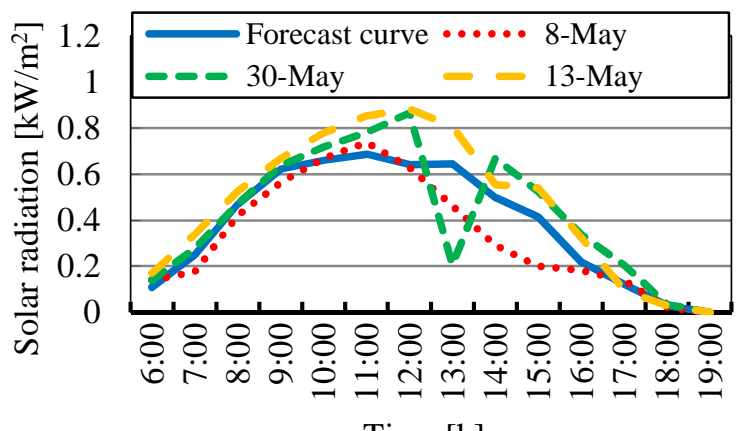

Time [h]

Fig. 7 Predicted value and the measured value for the weather pattern-4

Fig .6 shows that the prediction errors of solar radiation is generally small except for that of weather pattern-4 and the errors are small when the weather is cloudy, rainy or fine throughout the day. If the weather does not change throughout a day, we can select one of similar weather patterns of the past as the reference pattern for the prediction. By this selection of a past weather pattern, the errors between the predicted value and the measured value become so small.

In pattern-4, as fine weather suddenly turned to cloudy in the middle of the day, errors of the prediction are large. Hence, the errors of patterns between the predicted values and the measured values were so large.

In addition, the reduction of the number in the weather patterns from 15 to 6 achieved the improvement of the prediction error. By reducing the number of patterns, we are able to increase the number of data to work out the prediction curve and this leads to enhance the applicability of the proposed method to different areas and weather conditions.

Fig. 7 shows we can predict chnges of solar radiation curves for three days. This means that the accuracy of the prediciton can be improved with the consideration of the changes of solar radiation in sunrise and sunset. On the contrary, the proposed method could be predicted the rapid change of the radiation at 1:00 pm on May 30. The reason of this is that the correction of the prediction is conducted at 8:00 and therefore the sudden change of radiation at 1:00 pm is not taken into consideration in the current prediction. 


\subsection{Comparisons of errors before and after corrections in 6 weather patterns}

The previous section presented the improvement of errors by reducing the number of weather patterns. This section describes the improvement of errors between the prediction and that after the correction by using online measured data.

In Table 3, the predicted radiation curve on the previous day is corrected by observing online measured data on the targeted day and the errors between the predicted and corrected radiation curves are compared for 6 weather patterns. Fig. 8 and Fig. 9 show that the measured values, the predicted values and the corrected values by the proposed method in April and July.

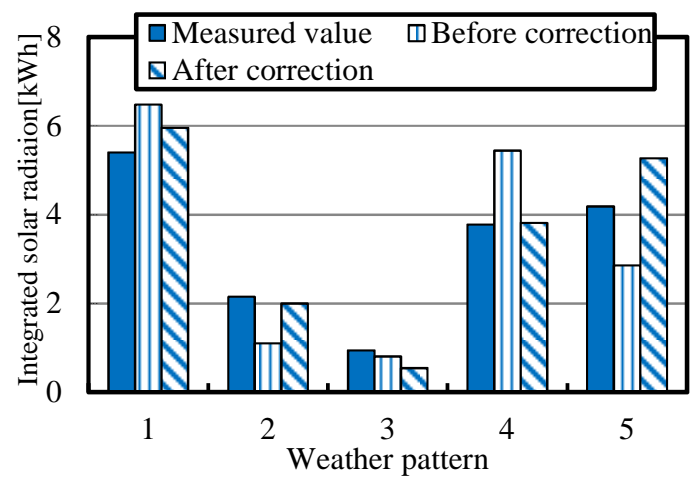

Fig. 8 Comparisons of the average accumulated solar radiation of each weather pattern in April

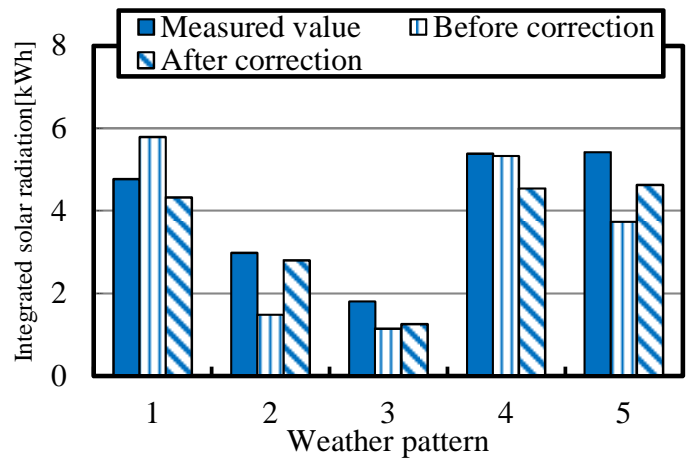

Fig. 9 Comparisons of the average accumulated solar radiation of each weather pattern in July

Fig. 8 and Fig. 9 show that the error for patterns 2 and 3 was decreased by the correction. As a result, the revisiones can be reduced the error of the accumulated solar radiation of the day by almost $34 \%$. Generally, the amount of the solar radiation almost unchanged on cloudy and rainy days and the radiation remains at the value close to the radiation at $8.00 \mathrm{am}$. Thereby, the tested results indicate that the proposed method has effectiveness of the curtailment of the errors. Lastly, for pattern-4, the measured radiation curve, the predicted curve, and the curve after the correction are shown in Fig. 10 and Fig. 11.

Fig. 10 shows that the proposed method is able to perform the better accurate prediction of the solar radiation, if the solar radiation is less than that of previous day. By the radiation curves in Fig. 11, it shows that the correction of the measured radiation improves the accuracy of the prediction even if the weather forecast is not correct.

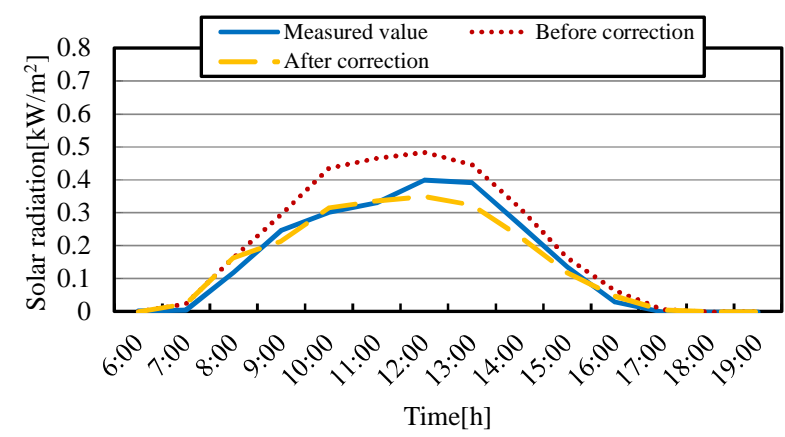

Fig. 10 Predicted and measured radiation on January 10,2012

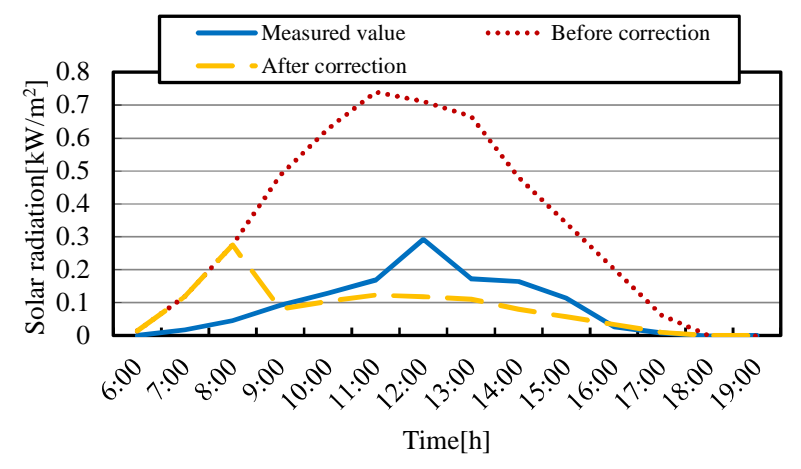

Fig. 11 Predicted and measured radiation on March 10,2012

\section{Future studies}

In this study, the author proposes the prediction method by dividing the prediction into two stages; predictions on the previous day and on the day. The correction was conducted by using the online measured data at 8:00. In the future, the correction is to conduct in different time zones and carry out repetitively. The predicted value can lead to a large error because this method does not take the unexpected weather variation into account. Therefore, it is necessary to conduct the analysis of correlation of the solar radiation and meteorological conditions, such as temperature, pressure and humidity. Then results of the correlation analysis are required to incorporate into the future prediction model. In addition, the number of the weather patterns is likely to change when the prediction is conducted in different areas. In the future, the author aims to increase the precision of the prediction to investigate the relationship between other meteorological elements and solar radiation.

\section{Conclusion}

In this study, the prediction of the solar radiation is divided into two stages; predictions on the previous day and on the day. The prediction for the previous day is conducted with the weather forecast and the weather patterns. As a result, the error is decreased by the reduction of the number of weather patterns. In addition, the error of the accumulated solar radiation is reduced by almost $34 \%$ by the prediction of the day. Furthermore, even the weather forecast of the previous day is not correct as expected, the error is reduced by the correction of the radiation curve on the day by measuring online radiation. 


\section{References}

[1] Shinichirou Orita,Yoshishige Kemmoku ,

Shigeyasu Nakagawa ,Tateki Sakakibara :'Insolation Prediction by a Multi-Stage Neural Network", T.IEE Japan,Vol.117-B,No.8,'97(in Japanese)

[2] Yoshishige Kemmoku,Shigeyasu Nakagawa,Teru Kawamoto,Tateki Sakakibara:"Operational Methods of a Solar-heat/Electricity Hot-water Suppy System Using the Insolation Forecast of Next Day",T.Iee Japan,Vol.119B,No.10,'99(in Jpanese)

[3] Takae Shimada,Kosuke Kurokawa:'Insolation Prediction Using Weather Forecast with Weather Change Patterns",IEEj Trans.PE,Vol.127,No.11,2007

[4] Susumu Yamashiro, Takayuki Koyama, Koichi Nakamura, Hirofumi Tezuka, Shigemitsu Kakizoe:"An Operating Method and Economic Estimation of Grid-connected Rsidential Photovoltaic Systems with Battery",T.IEE Japan,Vol.115-B,No.10,'95(in Japannese)

[5] Atsushi Yona, Kosuke Uchida, Tomonobu Senjyu, Toshihisa Funabashi”Optimal Planning Strategy for Large PV/Battery System Based on Long-Term Insolation Prediction",IEEJ Trans.EIS,Vol .131,No.10,2011(in Japannese)

[6] Kiyohumi Fujii Kaoru Koyanagi Ryuichi Yokoyama "Measurement and Analysis of Wind Speed and Intensity of Radiation for Feasibility Survey of Wind Power Generation and Photovoltaic Generation",PE-09-126,PSE09-134(in Japanese)

[7] Takashi Oozeki,Toshiyasu Izawa,Kenji Otani,Kosuke Kurokawa"An Estimation Method of Irradiation Data for Evaluating PV Systems",IEEJ Trans.PE,Vol.125,No.1,2005(in Japanese)

[8] Atsushi Yona, Tomonobu Senjyu, Ahmed Yousuf Saber, Toshihisa Funabashi, Hideomi Sekine, Chul-Hwan Kim"Application of Neural Network to 24-hour-Ahead Generating Power Forecasting for PV System"2008 IEEE(in English)

[9] Fumitoshi Nomiyama, Joji Asahi, Takuma Murakami, Hirotaka Takano, Junich Murata"A Study on Global Solar Radiation Forecasting Models Using Meteorological Data and their Application to Wide Area Forecast", 978-14673-2868-5/12\$31.00 2012 IEEE(in English)

\section{Biography}

Yosuke Kobayashi

was born in Aichi, Japan, on April 5,

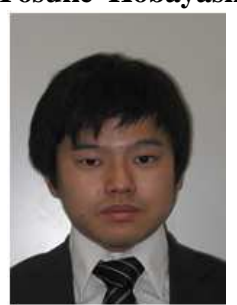
1988. He received B.S degree in electrical engineering from Hosei University, Tokyo, Japan, in 2012. He is now a graduate student at the Graduate School of Environment and Energy Engineering in Waseda University in Japan. His research interest is the prediction method of $\mathrm{PV}$ generation.

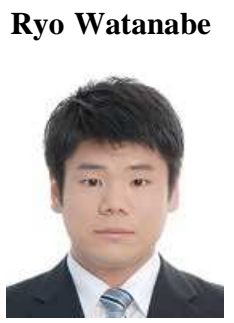

was born in kanagawa, Japan, on November 7, 1988. He received B.S degree in electrical engineering from Waseda University, Tokyo, Japan, in 2011. He is now a graduate student at the Graduate School of Environment and Energy Engineering in Waseda University in Japan. His research interest is PV generation's impact assessment on power systems.
Yusuke Hida was born in Kagoshima, Japan, on February 9, 1986. He graduated from department of electrical engineering in

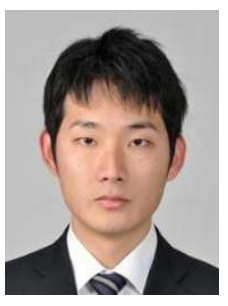

Meisei University, Tokyo Japan, in 2008. He received the degree of M.S in environment and energy engineering from Waseda University in 2010. He is now Ph.D student at the graduate school of environment and energy engineering in Waseda University in Japan. His research interests are environmental issues of power systems.

Ryuichi Yokoyama received the degrees of B.S., M.S. and Ph.D.

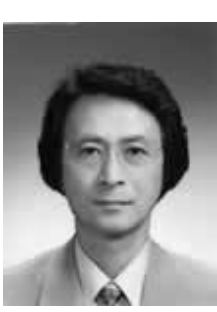
in electrical engineering from Waseda University, Tokyo Japan, in 1968, 1970 and 1973, respectively. After being engaged in Mitsubishi Research Institute, from 1978 through 2006, he worked in the Faculty of Technology of Tokyo Metropolitan University, and since 2007, he has been a professor at the Graduate School of Environment and Energy Engineering in Waseda University, Japan. Prof. Yokoyama is a fellow of IEEE of USA and the IEE of Japan and members of SICE of Japan, CIGRE, etc.

Toshihisa Funabashi (M'90, SM'96) was born in Aichi,

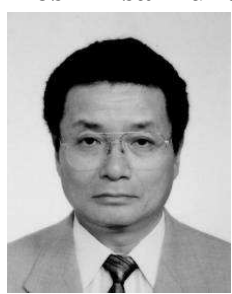
Japan, on March 25,1951. He graduated in March 1975 from the Department of Electrical Engineering, Nagoya University, Japan. He received, in March 2000, a Doctor degree from Doshisha University, Kyoto, Japan. He joined Meidensha Corporation in April 1975 and has generation. Currently, he is a Senior Engineer of the Power Systems Solutions Sales and Engineering Division. Dr. Funabashi is a chartered engineer in UK, a member of IET and the IEE of Japan. 